\title{
Crowd intelligence evolution based on complex network
}

\author{
Jianran Liu
}

Institute of Computing Technology, Chinese Academy of Sciences, Beijing, China and University of Chinese Academy of Sciences, Beijing, China, and

Wen Ji

Beijing Key Laboratory of Mobile Computing and Pervasive Device, Institute of Computing Technology, Chinese Academy of Sciences, Beijing, China

\begin{abstract}
Purpose - In recent years, with the increase in computing power, artificial intelligence can gradually be regarded as intelligent agents and interact with humans, this interactive network has become increasingly complex. Therefore, it is necessary to model and analyze this complex interactive network. This paper aims to model and demonstrate the evolution of crowd intelligence using visual complex networks.
\end{abstract}

Design/methodology/approach - This paper uses the complex network to model and observe the collaborative evolution behavior and self-organizing system of crowd intelligence.

Findings - The authors use the complex network to construct the cooperative behavior and self-organizing system in crowd intelligence. Determine the evolution mode of the node by constructing the interactive relationship between nodes and observe the global evolution state through the force layout.

Practical implications - The simulation results show that the state evolution map can effectively simulate the distribution, interaction and evolution of crowd intelligence through force layout and the intelligent agents' link mode the authors proposed.

Originality/value - Based on the complex network, this paper constructs the interactive behavior and organization system in crowd intelligence and visualizes the evolution process.

Keywords Crowd intelligence, Intelligence evolution, Complex network, Agent interaction,

Force layout

Paper type Research paper

\section{Introduction}

Intelligence is an important driving force for human development. With the vigorous development of artificial intelligence technology, intelligent technology has penetrated into many fields. The research on intelligence has once again pushed to a new height and no longer limited to computing science. Including human beings, anything that contains intelligence can be regarded as an agent with intelligence. These agents influence, interact

(C) Jianran Liu and Wen Ji. Published in International Journal of Crowd Science. Published by Emerald Publishing Limited. This article is published under the Creative Commons Attribution (CC BY 4.0) licence. Anyone may reproduce, distribute, translate and create derivative works of this article (for both commercial and non-commercial purposes), subject to full attribution to the original publication and authors. The full terms of this licence may be seen at http://creativecommons.org/licences/by/4.0/ legalcode

This work is supported by the National Key R\&D Program of China (2017YFB1400100) and the National Natural Science Foundation of China (62072440) and the Beijing Natural Science Foundation (4202072).

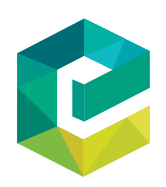

International Journal of Crowd Science 
IJCS

5,3

282

and evolve with each other to form a system of crowd intelligence. Crowd intelligence mainly explores ways to rationally use various intelligent resources, constructs the operation of intelligent body systems, taps the potential of intelligent interaction in specific fields and finally enhances benefits.

The crowd intelligence system contains multiple heterogeneous agents, these agents interact equivalently through the transformation from the subject space to the number space. The transformation from the subject space to the number space is called the intelligence measurement. After the measurement conversion, heterogeneous agents can associate and interact without obstacles. At this time, there are complex association relationships among individuals, which eventually form a network structure, known as the crowd intelligence network. With the passage of time, agents in the crowd intelligence network may have certain changes in spatial location, intelligence quantity and interaction ability. For example, the spatial distribution of interconnected agents should be denser, agents in the interaction process may also affect each other's intelligence. This kind of crowd intelligence network that changes over time is called the evolution of crowd intelligence.

Regarding the changes in space, distribution and amount of intelligence that exist in the evolution of the intelligence network, we propose to use complex network to describe the evolution of the intelligence network. First, we elaborated on the deployment process of the visualized complex network, including the visualized distribution, linking methods and parameter settings of agent nodes. After that, we constructed the interaction mode of individuals in the state-based network and pointed out the visual details of the evolution process. Finally, we designed the evolution experiment and the extreme case observation experiment, respectively. The purpose of this experiment is to verify the effectiveness of our proposed crowd intelligence network and its evolution. The experimental results show that the complex network we deployed for crowd intelligence's evolution can describe the evolution process of the crowd intelligence network in a visual distribution.

The contributions of this paper are summarized as:

- Complex networks are used to construct interaction models of crowd intelligence. Nodes and edges represent agents and interaction relationships, respectively.

- We design the way agents interact with each other. Neighboring agents interact to change their intelligence.

- The evolution of crowd intelligence in extreme cases is explored by analyzing the agent distribution of different intelligences.

The rest of the paper is organized as follows: Section 2 describes the work related to crowd intelligence and complex networks. In Section 3, we describe the complex network deployment and evolution mode of the evolution of crowd intelligence. Section 4 shows the evolution effect test and extreme evolution test results of the deployed crowd intelligence network. Finally, in Section 5, we summarize the whole work proposed and the prospect of future work.

\section{Related work}

\subsection{Crowd intelligence}

The concept of crowd intelligence was put forward with crowd computing [Miller (2012)] in the early days, emphasizing the importance of humlan beings in computing science. The current research on crowd intelligence focuses on the internal mechanism within the group. For example:

- To study how human and computer systems form a group of interoperable computing to complete complex tasks that are difficult for computer systems to complete independently [Yu et al. (2017)]. 
- How the heterogeneous system composed of man-machine-software can cooperate to achieve the optimal control mechanism [Shirado and Christakis (2017)].

- Introduce people into the system as experts to participate in processing, feedback and decision-making, forming a mixed man-machine system, so as to maximize the role of human knowledge in the application system [Ooi et al. (2014)].

The calculation and measurement of intelligence quantity of intelligent agents usually refer to the measurement method of human intelligence, the measurement of human intelligence depends on the determined computing system. The existing methods for human measurement usually adopt IQ tests, which is similar to methods for machine intelligence measurement. The core idea is the accuracy rate of completing certain kinds of questions in unit time. In recent years, the population entropy [Zhang et al. (2008)], comparative entropy [Peter (2010)] and potential field [Neil et al. (2018)] analysis which measure crowd intelligence behavior have provided good ideas. However, for heterogeneous agents, there is no clear standard for the transformation method from the main space to the digital space.

Similar to the inter-transformation between heterogeneous intelligences, there is no unified model for the evolutionary pattern of crowd intelligence. Because there are massive uncertain interactions in the real world, the relationship and degree of interaction may even change over time. In this paper, we model, regulate and observe the evolutionary network of crowd intelligence from a macroscopic perspective.

\subsection{Complex network}

The complex network theory is the result of observation of relatively simple network topologies in different fields. It is found that these networks are different from the previous network systems, they are dynamic and open, growing and evolving with the characteristics of life. At the local level, it is disorganized, while at the whole level, it is highly clustered. In particular, when the fields involved are different, it is necessary to integrate the knowledge of various related disciplines to establish the transformation from the subject space to the digital body space, so as to form a complex network belonging to this field. The research field of complex network mainly involves: cellular network [Zhang et al. (2016)], social network [Wang et al. (2019)], epidemic disease transmission network [Zhuang and Yagan (2020)] and so on.

At present, the research work of complex networks mainly focuses on the following aspects:

- Research on the model and theory of complex network [Zhou et al. (2009)]. It includes more extensive empirical research and more in-depth theoretical characterization, such as matching patterns based on given degree distribution, various correlation [Geng et al. (2018)], statistical properties and description of weighted network and network clustering [Lei et al. (2019), Ooi et al. (2014)], etc.

- Evolution and mechanism model of complex network [Bettencourt (2014)]. It mainly studies the statistical law of actual network evolution [Yang et al. (2018)] and theoretically can develop a more perfect network mechanism model with specific geometric properties.

- Structure of complex network [Liu et al. (2014)]. Functional and network dynamics research, including network fault tolerance and attack robustness [Shang (2017)], as well as network propagation [Karyotis and Papavassiliou (2015)], synchronization and resonance dynamics processes. 
IJCS

5,3

In this paper, combined with the characteristics of complex networks, the science of public intelligence and its evolution model are expressed in the form of complex networks. The next section describes the details of complex network construction that incorporates the features of crowd intelligence.

\section{Establishment of crowd intelligence distribution and complex network}

The two basic elements of a complex network are nodes and edges. In this paper, they are set as:

(1) Node: in our model, a node is used to represent an intelligent individual. The attributes of a node include the independent ID $i$ of the intelligent individual and the intelligence quantity $q_{i}$ of $i$. The set of all the $n$ nodes is represented as $\mathbf{N}=\left\{i_{1}, i_{2}, \cdots, i_{n}\right\}$. For each node $i$ in $\mathbf{N}$, it contains its position attributes $i_{x}$ and $i_{y}$ in coordinates.

(2) Edge: an edge represents an association between two agents. The properties of an edge include only two nodes linked by it. The set of all edges is represented as $\mathbf{E}=\{(i, j) \mid i, j \in \mathbf{N}, i \neq j\}$.

The whole crowd intelligence evolution map is represented as:

$$
\mathbf{G}=(\mathbf{N}, \mathbf{E})
$$

This section describes the layout process and evolution process of crowds respectively. It is worth noting that the next calculation involves taking pixels (Pix) as the unit of measurement.

\subsection{Layout process}

First, we exclude edges and consider the distribution of nodes only. Assuming that the number of summation nodes is $n$, the coordinates $x$ and $y$ of each node are uniformly distributed in the circular region $x^{2}+y^{2} \leq r^{2}$. Therefore, the distance between each node is almost equal. Figure 1 (a) shows the distribution of $n=150$ nodes in a circle with radius $r=720 \mathrm{Pix}$.

Next, we group $n$ nodes. The purpose of this is to make the resulting distribution have multiple populations and thus contain a variety of evolutionary environments. Suppose each group contains $g$ nodes, these $g$ nodes are assigned new IDs 1 to $g$ and participate in the edge establishment together. Base on Figure 1 (a), Figure 1 (b) shows the result of node linking. 150 nodes are divided into two groups, including 90 nodes and 60 nodes respectively. For the nodes $i_{g}$ and $j_{g}$ (make sure that $i_{g}>j_{g}$ to avoid repeated links) in a group with $g$ nodes, they are linked if and only if:

$$
d \frac{i_{g} j_{g}}{g^{2}}>\operatorname{rand}
$$

where rand is a random number with standard uniform distribution. Link density $d \in(0,1)$ is used to control the probability of association between nodes. Figure 1 (b) shows the case when $d=0.0625$. Under the constraint of $i_{g}, j_{g} \in(1, g)$, when $d=1$, the theoretical possibility of two nodes linking is 0.25 . According to the setting of 90 nodes as a group, the numbers of the two main populations formed in the graph are bounded by 90 . However, these linked nodes no longer obey the uniform distribution as shown in Figure 1 (a). The distribution of these nodes follows the Fruchterman-Reingold (FR) [Bi et al. (2018)] 


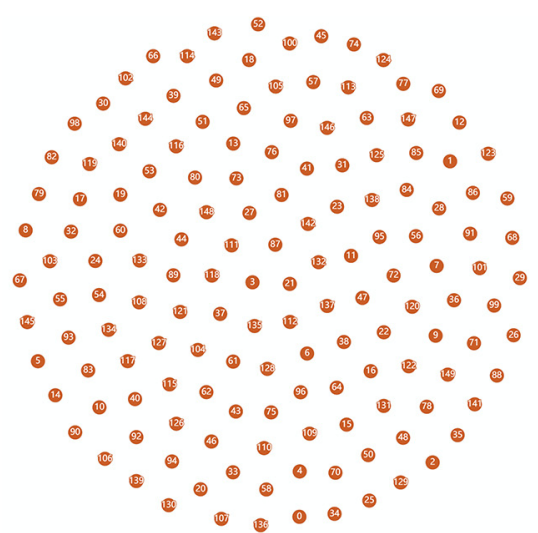

(a)

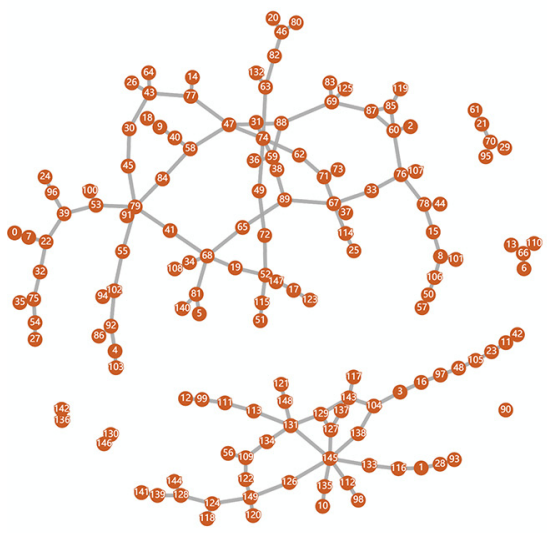

(c)

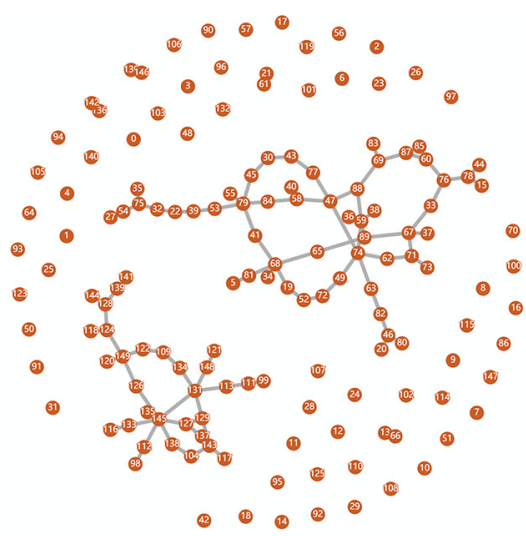

(b)

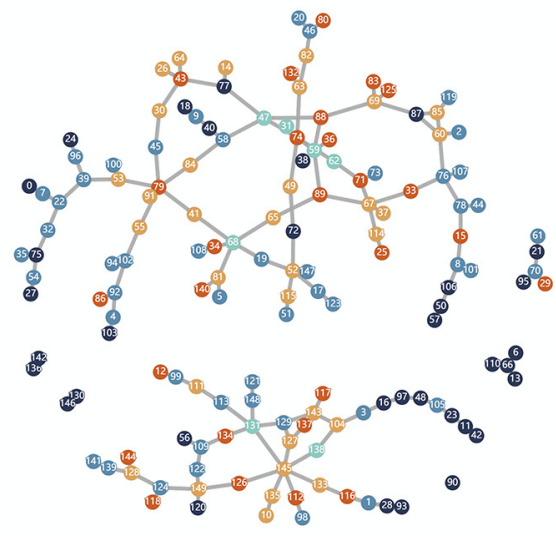

(d)
Figure 1. Different stages of the formation of the crowd intelligence network. It includes the initial uniform distribution pattern, the agent node linking pattern and the evolutionary distribution

algorithm. The FR algorithm treats each node as an electron in the nucleus and is affected by two forces: The coulomb force $f_{c}$ generated by other nodes and the gravitational force $f_{g}$ generated by linked nodes. These two forces are respectively expressed as:

$$
\left\{\begin{array}{r}
f_{c}=\frac{s^{2}}{c \sqrt{\frac{\pi r^{2}}{n}}} \\
f_{g}=\frac{-\frac{\pi r^{2} c^{2}}{n}}{s}
\end{array}\right.
$$

where $s$ represents the ideal distance between two points and $c$ stands for constant and is used to control the optimal distance between two nodes. For each node, its forces need to gradually reach a balance and finally form a stable distribution. It is worth noting that due 
IJCS

5,3

to the intervention of the FR method, the two unrelated crowds present an exclusive relationship in the distribution.

Then, for the nodes that are not linked in Figure 1 (b), we need to give these nodes a chance to link. Assuming that node $i_{u}$ with no edges is assigned, the ID of the target correlation node we assign to $i_{u}$ is:

$$
j=\left\lfloor\operatorname{rand}^{*}\left(n-i_{u}\right)\right\rfloor, j \in \mathbf{N} .
$$

Figure 1 (c) shows the result after adding edges. It can be seen that it is composed of two distinct large crowds and several other sporadic small crowds. The formation of two large crowds is the result of group division in the previous step.

\subsection{Evolutionary process}

After the whole crowd intelligence map $\mathbf{G}$ is established, the evolution process can be started. When constructing Figure 1 (a), our method randomly allocates intelligent quantities. Every element in $\mathbf{Q}$ follows the standard uniform distribution. The other two factors influencing the evolution of crowd intelligents are interaction rate $\alpha \in(0,0.5)$ and volatility rate $v \in(0,1)$. Interaction rate $\alpha$ is used to represent the degree of correlation between nodes, volatility rate $v$ is used to describe the possibility of uncertainty and degradation that may occur in the interaction process. In the evolution from generation $t$ to generation $t+1$, when two agents $i_{t}$ and $j_{t}$ interact with each other, their intelligence $q_{i}^{t}$ and $q_{j}^{t}$ are change as:

$$
\left\{\begin{array}{c}
q_{i}^{t+1}=\alpha_{t} q_{j}^{t}\left(\text { rand }-v_{t}\right) \\
q_{j}^{t+1}=\alpha_{t} q_{i}^{t+1}\left(\text { rand }-v_{t}\right),
\end{array}\right.
$$

where $i_{t}$ and $j_{t}$ belong to the asynchronous interaction pattern, so the intelligent increment of $q_{j}^{t}$ is related to $q_{i}^{t+1}$. In each evolutionary generation $t, \alpha_{t}$ and $v_{t}$ may be different. The state of the evolutionary network at the $t$ th evolutionary stage is described as:

$$
\mathbf{G}_{t}=\left(\mathbf{N}_{t}, \mathbf{E}, \mathbf{Q}_{t}, \alpha_{t}, v_{t}\right)
$$

where only the edge set $\mathbf{E}$ does not change with evolution. Although the number $n$ of nodes remains unchanged, there are many types of node positions that satisfy the FR algorithm. So the position of $\mathbf{N}$ at each moment changes, but the magnitude of this change is small. Figure 1 (d) shows the evolution of Figure 1 (c) at $t=60, \alpha_{1 \ldots 60}=0.1, v_{1 \ldots 60}=0.2$. Different colors of nodes represent different levels of intelligence. In the next subsection, we elaborate on some of the details of the evolution map that may influence our subsequent experimental analysis.

\subsection{Details}

Figure 2 zooms in on Figures 1 (d) to show more detail. There are three main types of details:

(1) Color distribution. Over the course of evolution, we've noticed an exponential increase in the intelligence of individuals. To better represent the process of evolution, we chose $4^{n}$ as the step size and divided five different levels of intelligence.

(2) No other crowds are allowed in the closed area. For example, in the closed circle composed by the nodes 69-88-59-62-71-67-33-76-60-87-69 in Figure 2, crowds like 142-136 are not allowed to be distributed in it, even if the FR algorithm allows it to happen. 


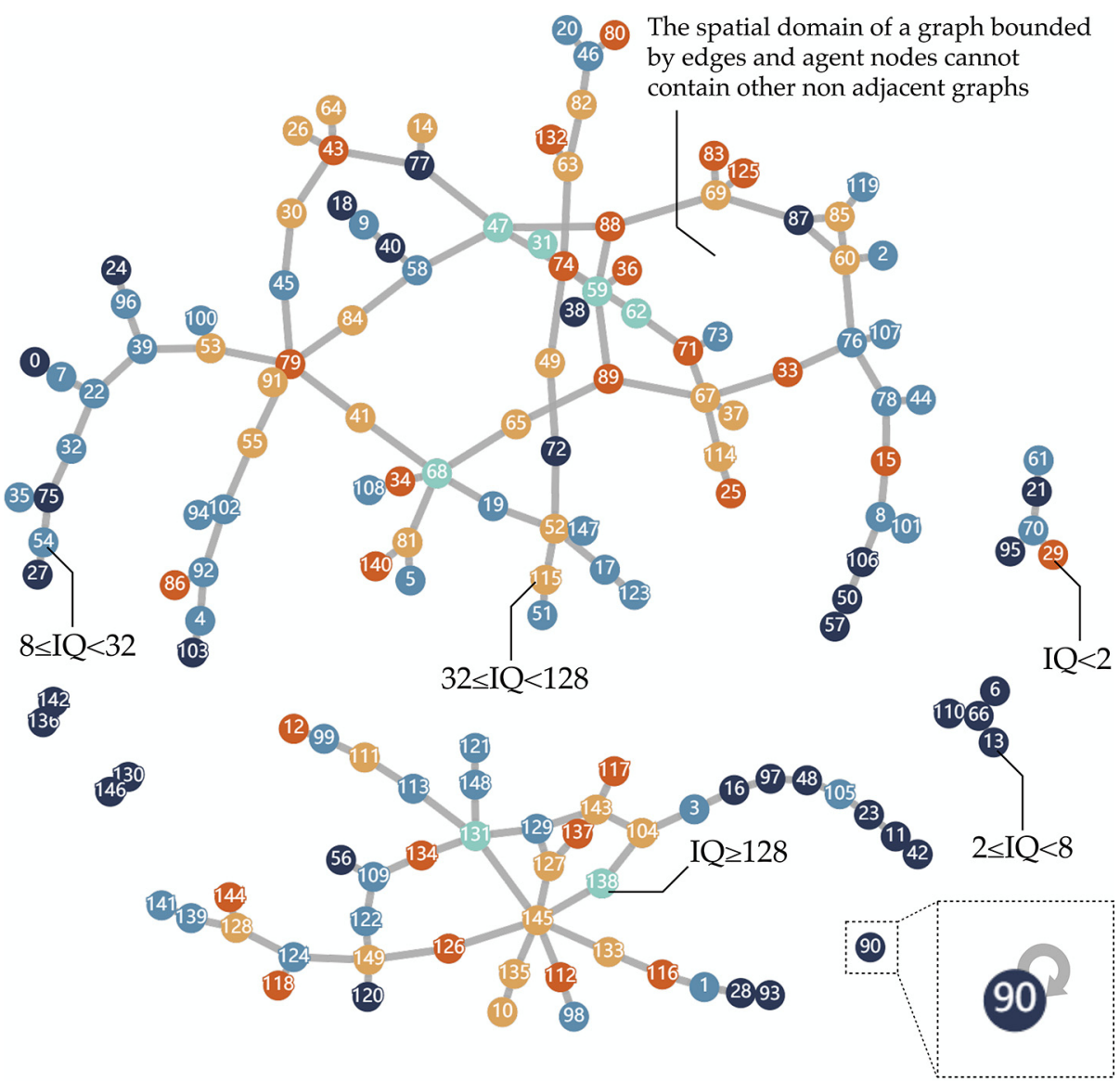

When an individual has no adjacent nodes, the default is self-association.
Figure 2.

Details in the visualization of evolutionary processes. This figure shows the colors corresponding to the range of agent intelligence. In particular, some nonnegligible distribution details and interaction details are also presented in this figure

(3) Self-connection. In our linking process, there may be free nodes. This kind of nodes is not without links, but they are linked to themselves (e.g. node 90 in Figure 2).

\section{Experiments and observations}

To verify the reliability of our proposed interaction complex network describing the evolution of crowd intelligence, we designed two different experiments: conventional evolution experiments and extreme environment evolution observations. The commonalities in the parameter settings of these two experiments are:

- $n \equiv 150$. Keep the number of samples per experiment constant.

- $g_{1} \equiv 90, g_{2} \equiv 60 . n$ nodes are divided into two groups, which are 90 and 60, respectively.

- $d \equiv 0.0625$. Link density is maintained at 0.0625 .

- The deployment of node locations follows the FR algorithm in the previous section. 
IJCS

5,3

288

\subsection{Conventional evolutionary experiments}

In the experiments, we first demonstrate the effect of changes in interaction rate $\alpha$ on the evolution of crowd intelligence. We start from 0.08 and set 3 sets of different interaction rates with 0.02 as the step size. Figure 3 shows the population intelligence distribution map at $t=60$. The line graph corresponding to each distribution map reflects the individual's intelligence status after each evolution. $\bar{Q}$ represents the average intelligence of all agents and $D_{Q}$ represents the variance of the intelligence of all agents.

\section{(a)}

$\alpha_{1 \cdots 60}=0.08$
$v_{1 \cdots 60}=0.2$

(b)

$\alpha_{1 \ldots 60}=0.1$

$v_{1 \ldots 60}=0.2$

(c)

Influence of different interaction rates on crowd intelligence evolution. In our proposed interaction method, even small changes in interaction rate can still produce qualitative changes after 60 evolutions
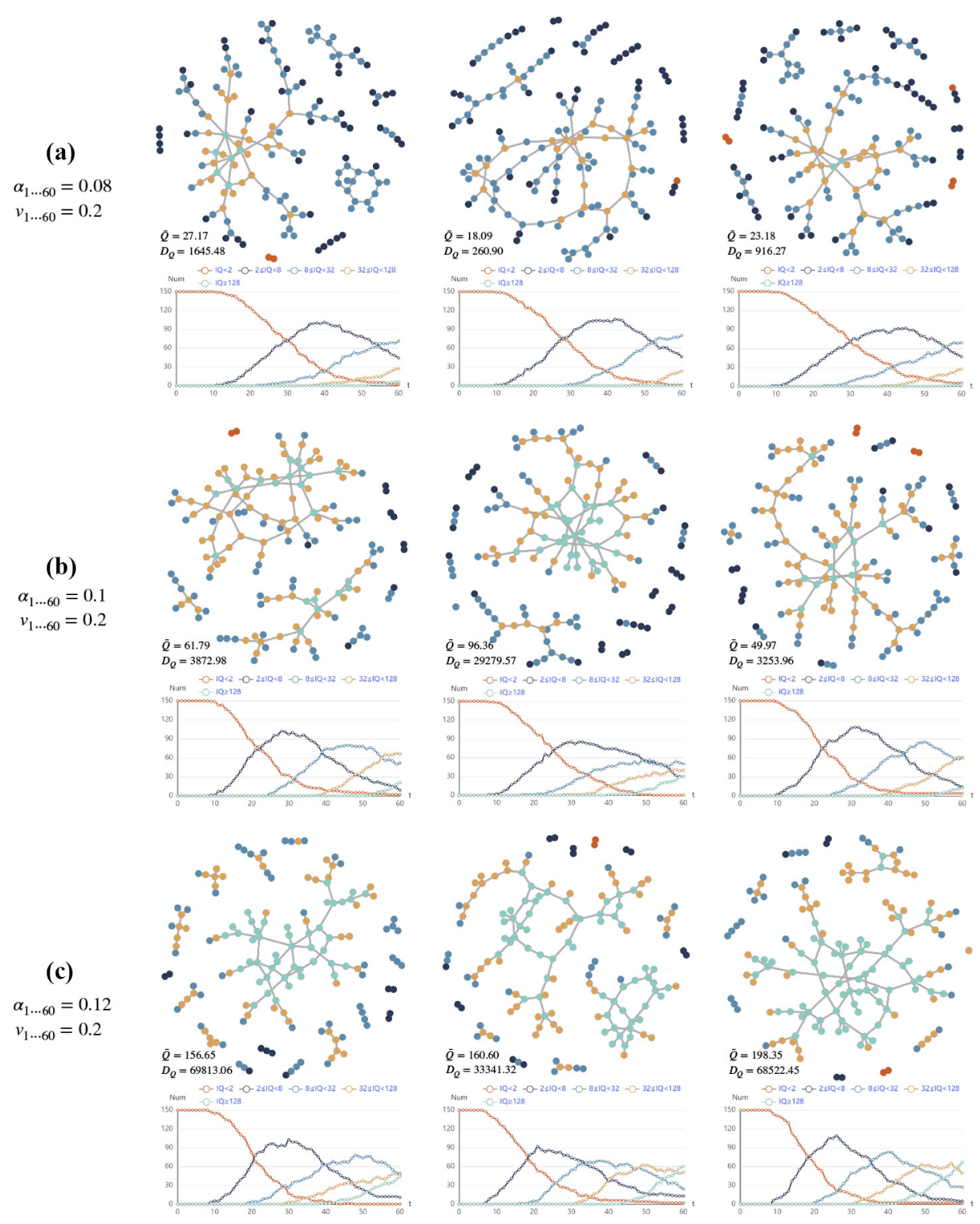
It can be seen from the evolution trend of different interaction rates in Figure 3 that the higher the interaction rate, the faster the evolution. In particular, the complex evolutionary network with $\alpha=0.12$ evolves into smarter individuals significantly earlier than the network with $\alpha=0.08$. In addition, as the intelligence of the agent increases exponentially, even a small-scale fine-tuning of the interaction rate. After 60 times of evolution, the increase in intelligence presents a butterfly effect.

On the other hand, we also designed an experiment to test the sensitivity of volatility rate. Figure 4 shows the evolutionary situations after adjusting for volatility rate at a fixed interaction rate. Comparing with Figure 3(b) we find that volatility rate will hinder evolution to a certain extent. The evolutionary uncertainty caused by volatility rate will affect the progress of evolution. The higher the volatility rate, the greater the obstacle to evolution.

However, there are two evolutionary phenomena worth noting:

(1) Normally, the node at the core of the interaction can first reach a higher level of intelligence in evolution (e.g. node 131 in Figure 2) and the node at the edge of the interaction or the end of the network usually evolves slowly.

(2) In the experiment shown in Figure 4, although the volatility rate of node interaction is adjusted, it still does not significantly interfere with the evolutionary trend. In the following extreme observations, we will focus on the impact of extreme volatility rate on evolution.

(a)

$\alpha_{1 \cdots 60}=0.1$

$v_{1 \ldots 60}=0.1$

(b)

$\alpha_{1 \ldots 60}=0.1$
$v_{1 \ldots 60}=0.3$
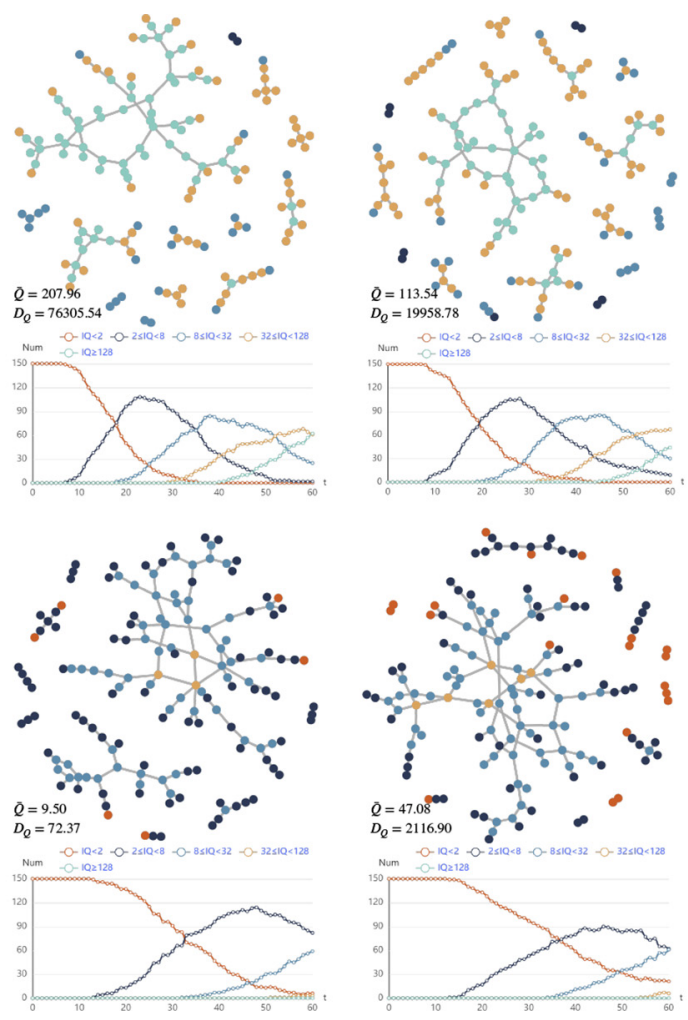
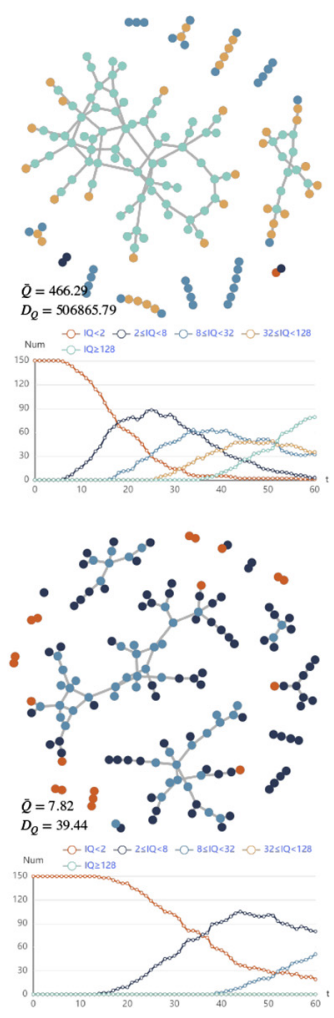

Figure 4.

Influence of different volatility rates on crowd intelligence evolution. In our volatility rate comparison experiment, although increasing volatility rate can stop the evolution of the wisdom of the crowd, it still fails to stop the trend of normal evolution 
IJCS

5,3

\section{Figure 5.}

Evolutionary distribution of wisdom under extreme volatility rate. The distribution on the left is the result of 120 evolutions with $\mathrm{v}=1$. Keeping the same distribution, we suddenly get volatility rate down to 0 and evolve it 60 times to get the distribution on the right. For different intelligence, we extract their distributions and measure the Kullback-Leibler divergence of them

\subsection{Observations of extreme cases}

When the volatility rate reaches a high level, it can block the trend of normal evolution. In the extreme situation experiment we designed, the interaction rate $\alpha$ is set to 0.2 and the volatility rate $v$ is set as 1 in the first 120 iterations. The left side of Figure 5 shows the

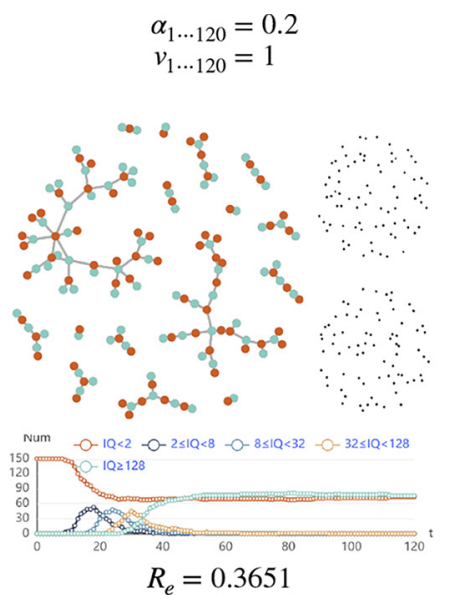

$$
\begin{aligned}
& \alpha_{1 \cdots 180}=0.2 \\
& v_{1 \cdots 120}=1, v_{121 \cdots 180}=0
\end{aligned}
$$
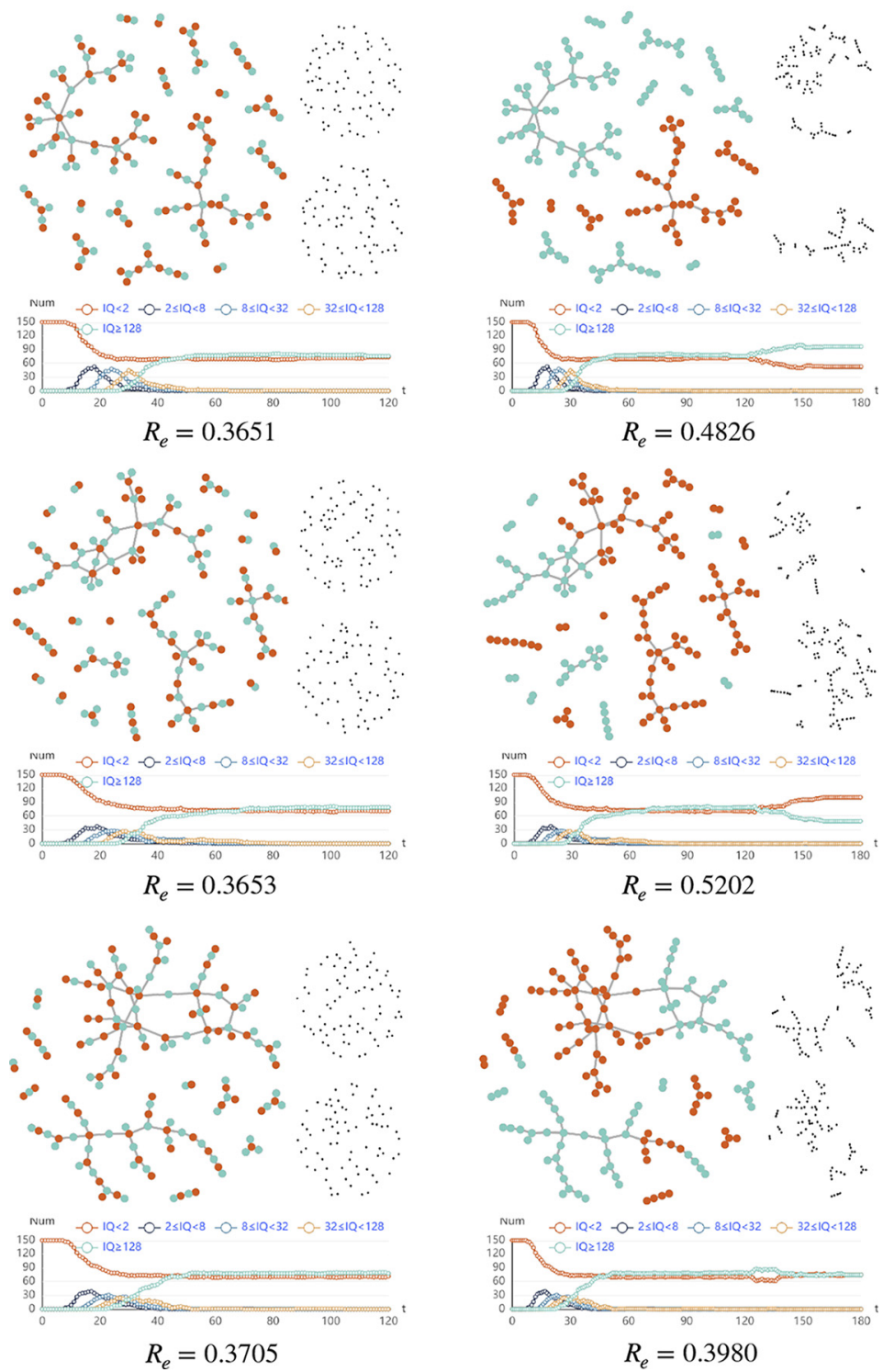
distribution and changes of crowd intelligence in this environment. In the first 50 iterations of evolution, the change of the agent is in a normal state. After 50 iterations of evolution, individuals with low intelligence and individuals with high intelligence gradually form a balance and are evenly distributed in space and almost converges. We extracted the distribution of low-intelligence individuals and high-intelligence individuals in the figure and calculated their Kullback-Leibler divergence $R_{\ell}$. Kullback-Leibler divergence is used to measure the distance between two distributions, the more similar the two distributions, the closer the Kullback-Leibler divergence between them is to 0 .

From the 121st round of evolution, we suddenly set the volatility rate to 0 and continue to evolve. The right side of Figure 5 shows the evolution state of the crowd intelligent after the volatility rate mutation. After continuing to evolve for 60 rounds, the entire distribution showed a new balance, the intelligent evolution curve also converged again after a small change. However, the low-intelligence individuals and high-intelligence individuals present a new distribution in the complex interactive network diagram and are different from the distribution at $t=120$. At $t=180$, These two types of individuals with different intelligence no longer have the same distribution and their Kullback-Leibler divergence is also increased compared with the previous state.

\section{Conclusion and future work}

The constant interaction of crowd intelligence leads to the evolution of agent intelligence, then, lead to the evolution of crowd intelligence. This paper describes the distribution, interaction and evolution of crowd intelligence using complex interactive networks. We describe in detail the organizational processes, interaction methods and parameters influencing evolution among agents. Specifically, we analyze evolution at a macro level by analyzing the distribution of agents with different intelligences. In the future, we will consider the variable interactivity of the network on the basis of the existing complex interactive network, so that the simulation of the complex network is closer to the real situation.

\section{References}

Bettencourt, L.M.A. (2014), "Impact of changing technology on the evolution of complex informational networks", Proceedings of the IEEE, Vol. 102 No. 12, pp. 1878-1891.

Bi, L., Wang, Y., Zhao, L., Qi, H. and Zhang, Y. (2018), "Social network information visualization based on Fruchterman-Reingold layout algorithm", IEEE 3rd International Conference on Big Data Analysis, pp. 270-273.

Geng, C., Qu, S., Xiao, Y., Wang, M., Shi, G., Lin, T., Xue, J. and Jia, Z. (2018), "Diffusion mechanism simulation of cloud manufacturing complex network based on cooperative game theory", Journal of Systems Engineering and Electronics, Vol. 29 No. 2, pp. 321-335.

Karyotis, V. and Papavassiliou, S. (2015), "Macroscopic malware propagation dynamics for complex networks with churn", IEEE Communications Letters, Vol. 19 No. 4, pp. 577-580.

Lei, M., Liu, L. and Wei, D. (2019), "An improved method for measuring the complexity in complex networks based on structure entropy”, IEEE Access, Vol. 7, pp. 159190-159198.

Liu, L., Liu, Y. and Zhang, N. (2014), "A complex network approach to topology control problem in underwater acoustic sensor networks", IEEE Transactions on Parallel and Distributed Systems, Vol. 25 No. 12, pp. 3046-3055.

Miller, R. (2012), "Crowd computing and human computation algorithm", Proceeding of the 2012 conference on ACMCI, pp. 1-2. 
IJCS

5,3

Neil, E., David, K. and Michael, B. (2018), "A new metric for the analysis of swarms using potential fields”, IEEE Access, Vol. 6, pp. 63258-63267.

Ooi, B.C., Tan, K.L., Tran, Q.T., Yip, J.W.L., Chen, G. and Ling, Z.J. (2014), “Application of differential evolution algorithm for transient stability constrained optimal power flow", Acm Sigkdd Explorations Newsletter, Vol. 16 No. 1, pp. 39-46.

Peter, C. (2010), “A measure of machine intelligence”, Proceedings of the IEEE, Vol. 98 No. 9, pp. 1543-1545.

Shang, Y. (2017), "Subgraph robustness of complex networks under attacks", IEEE Transactions on Systems, Man and Cybernetics: Systems, Vol. 49 No. 4, pp. 821-832.

Shirado, H. and Christakis, A. (2017), "Locally noisy autonomous agents improve global human coordination in network experiments", Nature, Vol. 5, pp. 370-374.

Wang, C., Koh, J.M., Cheong, K.H. and Xie, N. (2019), "Progressive information polarization in a complex-network entropic social dynamics model”, IEEE Access, Vol. 7, pp. 35394-35404.

Yang, Y., Li, J., Shen, D., Nan, M. and Cui, Q. (2018), "Evolutionary dynamics analysis of complex network with fusion nodes and overlap edges", Journal of Systems Engineering and Electronics, Vol. 29 No. 3, pp. 549-559.

Yu, C., Chai, Y. and Liu, Y. (2017), “Collective intelligence: from the enlightenment to the crowd science”, Proceeding of the 2017 conference on crowd science and engineering, pp. 111-115.

Zhang, G., Quek, T.Q.S., Huang, A. and Shan, H. (2016), "Delay and reliability tradeoffs in heterogeneous cellular networks", IEEE Transactions on Wireless Communications, Vol. 15 No. 2, pp. 1101-1113.

Zhang, L., Liang, C. and Lu, Q. (2008), "A novel small-population genetic algorithm based on adaptive mutation and population entropy sampling", Proceedings of 2008 7th World Congress on Intelligent Control and Automation, pp. 8738-8742.

Zhou, J., Yu, W., Li, X., Small, M. and Lu, L. (2009), "Identifying the topology of a coupled FitzhughNagumo neurobiological network via a pinning mechanism”, IEEE Transactions on Neural Networks, Vol. 20 No. 10, pp. 1679-1684.

Zhuang, Y. and Yagan, O. (2020), "Multistage complex contagions in random multiplex networks", IEEE Transactions on Control of Network Systems, Vol. 7 No. 1, pp. 410-421.

\section{Further reading}

Joveini, M.B.Z. and Sadri, J. (2018), "Application of fractal theory on motifs countiPlease provide the exact wording you wish to include as an acknowledgement of funding:ng in biological networks", IEEE/ACM Transactions on Computational Biology and Bioinformatics, Vol. 15 No. 2, pp. 613-623.

\section{Corresponding author}

Wen Ji can be contacted at: jiwen@ict.ac.cn

For instructions on how to order reprints of this article, please visit our website: 